\title{
Step Edges in Thin Films of Lamellar-Forming Diblock Copolymer
}

\author{
P. Stasiak, ${ }^{\dagger}$ J. D. McGraw, ${ }^{\ddagger}$ K. Dalnoki-Veress, ${ }^{\ddagger}$ and M. W. Matsen* ${ }^{\dagger}$ \\ ${ }^{\dagger}$ School of Mathematical and Physical Sciences, University of Reading, Whiteknights, Reading RG6 6AX, U.K. \\ ${ }^{\ddagger}$ Department of Physics \& Astronomy and the Brockhouse Institute for Materials Research, McMaster University, Hamilton, Ontario, \\ Canada
}

ABSTRACT: Self-consistent field theory (SCFT) is used to study the step edges that occur in thin films of lamellar-forming diblock copolymer, when the surfaces each have an affinity for one of the polymer components. We examine film morphologies consisting of a stack of $\nu$ continuous monolayers and one semi-infinite bilayer, the edge of which creates the step. The line tension of each step morphology is evaluated and phase diagrams are constructed showing the conditions under which the various morphologies are stable. The predicted behavior is then compared to experiment. Interestingly, our atomic force microscopy (AFM) images of terraced films reveal a distinct change in the character of the steps with increasing $\nu$, which is qualitatively consistent with our SCFT phase diagrams. Direct quantitative comparisons are not possible because the SCFT is not yet able to probe the large polymer/air surface tensions characteristic of experiment.

\section{INTRODUCTION}

The behavior of block copolymer melts on solid substrates has received considerable attention over the last two decades motivated by a wide range of applications. ${ }^{1-3}$ Not surprisingly, the earliest work ${ }^{4-8}$ involved the simplest conceivable system, thin films of symmetric $\mathrm{AB}$ diblock copolymer on flat substrates. In the bulk, symmetric diblock copolymer forms a lamellar phase of alternating A- and B-rich domains, where each period, $L_{0}$, consists of two monolayers. The main effect of confining the diblock copolymer to a thin film is to orient the domains. When the substrate and air surfaces of the film each have an affinity for one of the polymer components, the lamellae orient in the plane of the film, thereby forming a parallel lamellar $\left(\mathrm{L}_{\nu}^{\|}\right)$phase consisting of $\nu$ diblock monolayers. Because of the strong tendency to maintain the preferred domain size of the bulk, the film height is quantized, $H \approx L_{0} \nu$ / 2. Even values of $\nu$ are selected when the two surfaces have an affinity for the same component, and odd values occur when the two surfaces have opposite affinities. Usually the amount of material cast on the substrate is incommensurate with the preferred domain size, which leads to macrophase separation into thin and thick regions (i.e., terraces) separated by steps of height $L_{0}$. The topography of the air surface changes from islands to a bicontinuous network to holes as the area of the thicker region increases. ${ }^{4}$ The behavior becomes particularly interesting with the formation of elaborate terraced structures, when droplets $^{9-11}$ or rings ${ }^{12}$ of diblock copolymer are deposited on the substrate.

The presence of step edges separating different film thicknesses is ubiquitous in block copolymer films, and yet they have received relatively little theoretical attention. A step edge between the $\mathrm{L}_{\nu}^{\|}$and $\mathrm{L}_{\nu+2}^{\|}$phases will typically involve $\nu$ continuous monolayers plus one semi-infinite bilayer as depicted schematically in Figure 1. Ausserré et al. ${ }^{5}$ initially argued that the bilayer should exist one monolayer below the

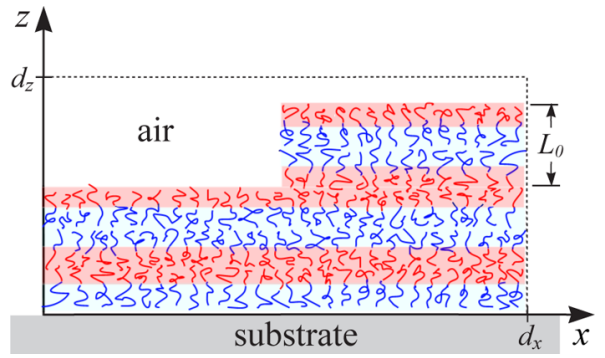

Figure 1. Schematic diagram of a step edge separating the $L_{\nu}^{\|}$and $L_{\nu+2}^{\|}$ phases, formed by $\nu=3$ continuous monolayers with a semi-infinite bilayer on top.

air surface, rather than on top as in Figure 1. Furthermore, they suggested that the lateral width of the step should be comparable to the bilayer thickness, $L_{0}$, but AFM measurements by Maaloum et al. ${ }^{13}$ found the steps to be much broader. Using an elastic-distortion model developed by Pershan ${ }^{14}$ for smectic-A liquid crystals, they were able to fit the surface profile of the steps. However, the elastic coefficients of the fit were inconsistent with estimates derived from block copolymer theory, ${ }^{15}$ which was attributed to the fact that the model neglects the polymer/air surface tension, $\gamma$. By employing a more general model ${ }^{16}$ that includes the surface tension, Turner et al. ${ }^{19}$ were able to obtain good fits with realistic coefficients. They also predicted that large surface tensions would force the semi-infinite bilayer toward the middle of the film, which was consistent with cross-sectional TEM images. However, further interrogation of TEM images by Liu et al. ${ }^{18}$ revealed a deficiency in the ability of the model to predict the shape of the

Received: October 12, 2012

Revised: November 16, 2012

Published: November 28, 2012 
internal interfaces. This was attributed to the fact that the model assumed a continuum of interfaces, which is inappropriate for thin films of just a few monolayers. Liu et al. attempted to overcome this deficiency by using an analytical block copolymer theory, ${ }^{15}$ which balanced the interfacial energy against the entropic stretching energy of the polymer chains within the discrete domains, but the complexity of the domain structure limited their progress.

Numerical self-consistent field theory (SCFT) overcomes this problem, and has been very successful in modeling the complex periodic morphologies observed in the bulk. ${ }^{19}$ This success has also been extended to thin films of uniform thickness, where the location of the diblock copolymer is constrained by a mask. ${ }^{20}$ The calculations can still predict the coexistence between different film thicknesses by performing a double-tangent construction, but they are not able to investigate step edges where the air surface needs the freedom to adjust its shape. To circumvent this problem, one could supplement the SCFT with an equation-of-state that allows it to handle large variations in the polymer concentration, ${ }^{21}$ but this is an unnecessary complication. Previous studies have avoided the need for this by simply replacing the air with a structureless fluid that creates an interfacial tension with the copolymer but has no other influence on the shape of the free surface. The filler is often chosen to be an incompatible solvent, ${ }^{22,23}$ but it can also be a homopolymer. ${ }^{24}$ Here we employ the latter approach to study the step edges between the parallel lamellar $\left(\mathrm{L}_{\nu}^{\|}\right)$phases of a thin film. We conclude by using the SCFT predictions to explain an intriguing experimental result, where AFM images reveal a distinctive change in the step edge as the number of monolayers is increased.

\section{THEORY}

This section describes the $\mathrm{SCFT}^{19,25,26}$ for a system of temperature $T$ and volume $V$ occupied by $n$ identical $\mathrm{AB}$ diblock copolymers, where the A and B blocks consist of $f N$ and $(1-f) N$ segments, respectively. For simplicity, we assume that all segments have the same statistical length, $a$, and each consume a volume, $\rho_{0}{ }^{-1}$, such that the copolymer fills a fixed fraction of the system, $\phi=n N / \rho_{0} V<1$. To prevent the copolymer from spreading into the air region, it is filled with a single incompatible homopolymer molecule of polymerization index $N_{\mathrm{h}}=(1-\phi) \rho_{0} V \gg N$ (the label h is used to denote homopolymer). We prefer to use a large homopolymer over the previous practice of using solvent, ${ }^{22,23}$ because this rids the filler of translational entropy which helps prevent it from swelling the copolymer film. To implement the mean-field approximation of SCFT, the molecular interactions acting on $\gamma$-type segments $(\gamma=\mathrm{A}, \mathrm{B}$ or $\mathrm{h})$ are replaced by external fields,

$$
w_{\gamma}(\mathbf{r})=N \sum_{\delta \neq \gamma} \chi_{\gamma \delta} \phi_{\delta}(\mathbf{r})+\xi(\mathbf{r})
$$

where $\phi_{\delta}(\mathbf{r})$ is a dimensionless concentration of $\delta$-type segments and $\chi_{\gamma \delta}$ is a Flory-Huggins parameter specifying the strength of the interaction between $\delta$ - and $\gamma$-type segments. The pressure field, $\xi(\mathbf{r})$, enforces the incompressibility condition, $\phi_{\mathrm{A}}(\mathbf{r})+\phi_{\mathrm{B}}(\mathbf{r})+\phi_{\mathrm{h}}(\mathbf{r})=1$.

The concentrations of the $\mathrm{A}$ and $\mathrm{B}$ segments are evaluated by

$$
\phi_{\mathrm{A}}(\mathbf{r})=\frac{\phi V}{Q} \int_{0}^{f} q(\mathbf{r}, s) q^{\dagger}(\mathbf{r}, s) \mathrm{d} s
$$

$$
\phi_{\mathrm{B}}(\mathbf{r})=\frac{\phi V}{Q} \int_{f}^{1} q(\mathbf{r}, s) q^{\dagger}(\mathbf{r}, s) \mathrm{d} s
$$

where

$$
Q=\int q(\mathbf{r}, s) q^{\dagger}(\mathbf{r}, s) \mathrm{d} \mathbf{r}
$$

is the partition function for a single copolymer in the external fields. The function $q(\mathbf{r}, s)$ is a partial partition function for the first $s N$ segments of the molecule, which satisfies the modified diffusion equation,

$$
\frac{\partial}{\partial s} q(\mathbf{r}, s)=\left[\frac{a^{2} N}{6} \nabla^{2}-w_{\gamma}(\mathbf{r})\right] q(\mathbf{r}, s)
$$

with the initial condition $q(\mathbf{r}, 0)=1$, where $\gamma=\mathrm{A}$ for $s<f$ and $\gamma$ $=\mathrm{B}$ for $s>f$. The other partial partition function, $q^{\dagger}(\mathbf{r}, s)$, for the last $(1-s) N$ segments satisfies the same diffusion equation, but with one side multiplied by -1 , combined with the condition $q^{\dagger}(\mathbf{r}, 1)=1$.

The analogous calculation for the concentration of homopolymer can be simplified by the ground-state dominance approximation $^{25,27}$ on account of its large polymerization. In the limit of large $s$, the partial partition function for the homopolymer becomes $q_{\mathrm{h}}(\mathbf{r}, s)=c \exp (-\lambda s) \psi(\mathbf{r})$, where $c$ is a constant, $\lambda$ is the smallest eigenvalue satisfying

$$
\left[\frac{a^{2} N}{6} \nabla^{2}-w_{\mathrm{h}}(\mathbf{r})\right] \psi(\mathbf{r})=-\lambda \psi(\mathbf{r})
$$

and $\psi(\mathbf{r})$ is the corresponding eigenfunction normalized such that $\int \psi^{2}(\mathbf{r}) d \mathbf{r}=V$. In this limit, the full partition function of the homopolymer reduces to $Q_{\mathrm{h}}=V c^{2} \exp \left(-\lambda N_{\mathrm{h}} / N\right)$ and its concentration is given by

$$
\phi_{\mathrm{h}}(\mathbf{r})=(1-\phi) \psi^{2}(\mathbf{r})
$$

The diffusion equations for $q(\mathbf{r}, s)$ and $q^{\dagger}(\mathbf{r}, s)$ are solved numerically in a $d_{x} \times d_{z}$ box with reflecting boundaries (see Figure 1), using the fourth-order pseudospectral method of Ranjan et al. ${ }^{28,29}$ with a spatial resolution of $\Delta x=\Delta z=$ $0.05 a N^{1 / 2}$ and a step size of $\Delta s=0.01$ along the polymer backbone. The eigenfunction, $\psi(\mathbf{r})$, is obtained by iterating the diffusion equation for $q_{\mathrm{h}}(\mathbf{r}, s)$ until its shape becomes independent of $s$, which typically occurs by $s=4$. Once $\psi(\mathbf{r})$ is known, $\lambda$ follows immediately from eq 6 . The self-consistency conditions are solved by applying simple mixing followed by Anderson mixing. ${ }^{30}$ The Anderson mixing scheme is usually used with a mixing parameter of $\lambda=1,{ }^{29,31,32}$ but here we had to reduce it to $\lambda=0.01$ in order to prevent the iterations from becoming unstable. This slows the rate of convergence, but we still obtain accurate solutions in $\sim 1000$ iterations.

There will generally be a number of distinct morphologies of the step edge, or rather self-consistent solutions of the field equations. The stable morphology is the one with the lowest free energy, $F$, which we evaluate using

$$
\begin{aligned}
\frac{N F}{k_{\mathrm{B}} T \rho_{0} V}= & -\phi\left[\ln \left(\frac{Q}{\phi V}\right)-1\right]+(1-\phi) \lambda \\
& +\sum_{\gamma} \frac{1}{V} \int\left[\frac{N}{2} \sum_{\delta \neq \gamma} \chi_{\gamma \delta} \phi_{\delta}(\mathbf{r})-w_{\gamma}(\mathbf{r})\right] \phi_{\gamma}(\mathbf{r}) \mathrm{d} \mathbf{r}
\end{aligned}
$$


Note that the free energy comparison must be performed using fixed values of $d_{x}, d_{z}$, and $\phi$.

\section{EXPERIMENT}

Step edges were prepared by depositing symmetric diblock copolymers onto a $\mathrm{Si}$ substrate with the native oxide layer. The molecules were poly(styrene-methylmethacrylate) (PS-PMMA) with total molecular weight $46 \mathrm{~kg} / \mathrm{mol}$ and a polydispersity index of 1.05 (Polymer Source Inc. Dorval, Canada). The silicon oxide surface has a strong affinity for the PMMA block while the air surface has a slight affinity for PS. We followed two established procedures for creating the films. The first ${ }^{12}$ produces films with a gradual variation in thickness, which after several hours of annealing order into parallel lamellae with a series of terraces. The second ${ }^{33}$ produces films of uniform thickness, which after annealing develop the usual island/hole formation with well isolated step edges. In both cases, the height of the individual steps closely matches the bulk lamellar period, $L_{0}=30 \mathrm{~nm}$, as observed from AFM scans across the surface.

\section{RESULTS}

Before performing a SCFT calculation for a step edge between the $L_{\nu}^{\|}$and $L_{\nu+2}^{\|}$phases, we have to first select a sufficiently large system. The width is set to $d_{x}=16 a N^{1 / 2}$, such that the effect of the step vanishes at the two boundaries, $x=0$ and $d_{x}$. Our choice for the vertical height, $d_{z}$, depends on the film thickness, $\nu$, but it is always large enough that copolymer concentration is essentially zero at $z=d_{z}$. The quantity of copolymer, $\phi$, is chosen to form a given number, $\nu$, of complete monolayers plus a half bilayer, such that the step occurs above the middle of the substrate (i.e., $x \approx d_{x} / 2$ ).

The behavior of the step edge is then controlled by just four quantities, $f, \chi_{\mathrm{A} B} N, \chi_{\mathrm{A} h} N$ and $\Delta \chi N$, where $\Delta \chi \equiv \chi_{\mathrm{Bh}}-\chi_{\mathrm{Ah}}$ dictates the affinity of the air surface. Without loss of generality, we assume $\Delta \chi>0$ such that the A component is favored at the air surface. The substrate is assumed to have a strong affinity for one of the components, but there is no need for a parameter specifying the strength of this preference. The affinity is accounted for by simply limiting our attention to morphologies that only have one component in contact with the substrate, which is achieved by having one of the continuous monolayers next to the substrate. ${ }^{34}$ Still, four parameters is a lot, and so we reduce the parameter space to a more manageable level by fixing the segregation of the diblock copolymer at an intermediate level, $\chi_{\mathrm{AB}} N=20$, for which the domains are reasonably pure. The surface tension between $\mathrm{A}$ and air is also held fixed by setting $\chi_{\mathrm{Ah}} N=30$.

Morphologies and Phase Diagrams. We begin our study by considering a step edge between the $L_{1}^{\|}$and $L_{3}^{\|}$phases. In this case, the film just has one continuous monolayer, which needs to be next to the substrate as explained above. With the semi-infinite bilayer confined to the top of the film, we investigate whether or not the edge of the bilayer core is exposed to the air. The top panel of Figure 2 shows the step edge for symmetric, $f=0.5$, diblock copolymer at a small surface affinity of $\Delta \chi N=10$, where the B-rich (blue) core is exposed to air (white); we label this as the open (O) morphology. The lower panel shows the step for a larger affinity, $\Delta \chi N=30$, where the top A-rich (red) domains of the $\mathrm{L}_{1}^{\|}$and $\mathrm{L}_{3}^{\|}$phases connect with each other to cover the edge of the bilayer core; we refer to this as a closed $\left(\mathrm{C}_{0}\right)$ morphology. Notice that the closure is aided by the edge of the core tilting slightly into the film, which causes a visible narrowing of the two domains below it.
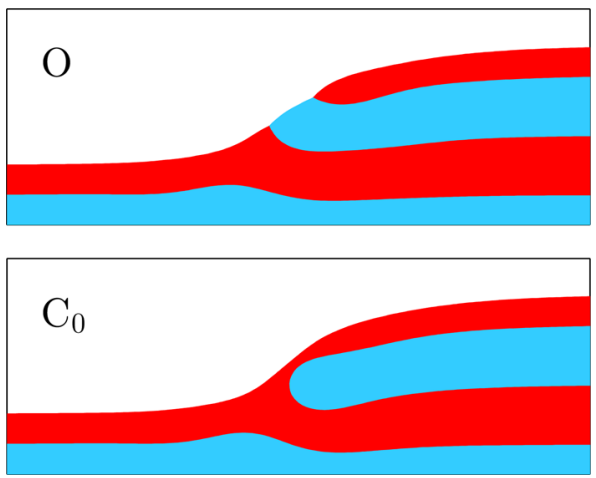

Figure 2. Open $(\mathrm{O})$ and closed $\left(\mathrm{C}_{0}\right)$ morphologies of the step edge separating the $L_{1}^{\|}$and $L_{3}^{\|}$phases, calculated for symmetric, $f=0.5$, diblock copolymers with surface affinities of $\Delta \chi N=10$ and 30, respectively. Each image is $8 \times 3$ in units of $a N^{1 / 2}$, and the domains are defined based on which component (A, red; B, blue; or h, white) has the highest concentration.

The phase diagram in Figure 3 shows the parameter regions over which the $\mathrm{O}$ and $\mathrm{C}_{0}$ morphologies occur. The curve

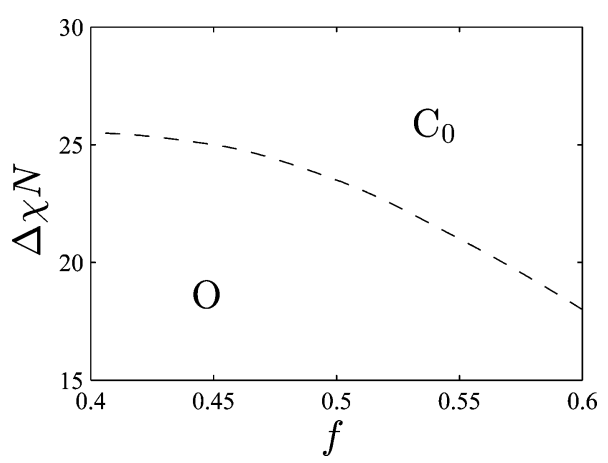

Figure 3. Phase diagram for the step edge separating the $L_{1}^{\|}$and $L_{3}^{\|}$ phases, showing the parameter regions over which the open $(\mathrm{O})$ and closed $\left(\mathrm{C}_{0}\right)$ morphologies in Figure 2 occur. The dashed curve denotes that the transition between them is continuous.

separating them is dashed to denote that the transformation from $\mathrm{O}$ to $\mathrm{C}_{0}$ occurs continuously, with the area of $\mathrm{B}$ in contact with air gradually vanishing as $\Delta \chi N$ increases. However, it is not a true thermodynamic phase transition, because it does not involve a singularity in the free energy. Indeed, its location is somewhat sensitive to how the domains are defined, which in our case is based on the component $(\gamma=\mathrm{A}, \mathrm{B}$ or $\mathrm{h})$ with the largest concentration, $\phi_{\gamma}(\mathbf{r})$.

Next we consider a step edge between the $L_{2}^{\|}$and $L_{4}^{\|}$phases. With the additional monolayer, there are now two possible closed morphologies, $C_{0}$ and $C_{1}$, as illustrated in Figure 4; the subscript denotes the number of complete monolayers above the semi-infinite bilayer. Note that the $C_{2}$ morphology is not considered, because one of the continuous monolayers has to remain beneath the semi-infinite bilayer on account of the strong affinity of the substrate. ${ }^{34}$

The phase diagram in Figure 5 displays the parameter regions where the different $L_{2}^{\|}-L_{4}^{\|}$step edge morphologies are stable. The open $(\mathrm{O})$ and closed $\left(\mathrm{C}_{0}\right)$ morphologies are again separated by a continuous transition, but it now happens at a weaker surface affinity. For $f=0.5$ diblocks, the transition from $\mathrm{O}$ to $\mathrm{C}_{0}$ occurs at $\Delta \chi N=22.0$, as compared to $\Delta \chi N=23.4$ in Figure 3. This is because the thicker film allows the B-rich core 

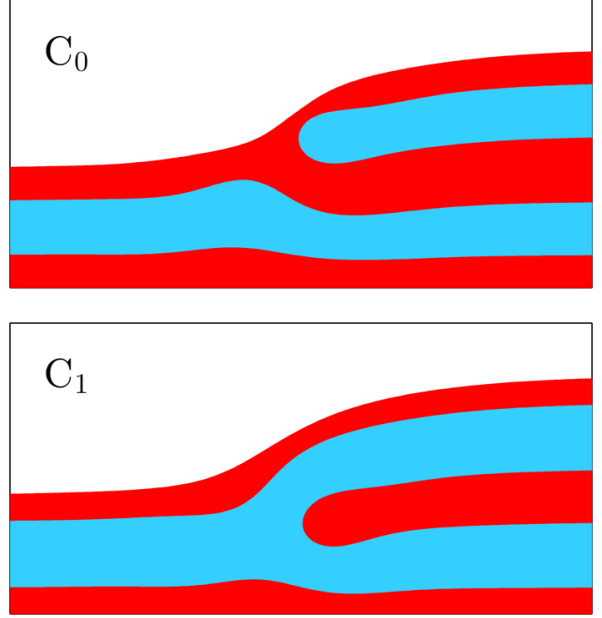

Figure 4. Closed $\mathrm{C}_{0}$ and $\mathrm{C}_{1}$ morphologies of the step edge separating the $\mathrm{L}_{2}^{\|}$and $\mathrm{L}_{4}^{\|}$phases, calculated for $\Delta \chi N=30$ with $f=0.55$ and 0.45 , respectively. Each image is $8 \times 4$ in units of $a N^{1 / 2}$.

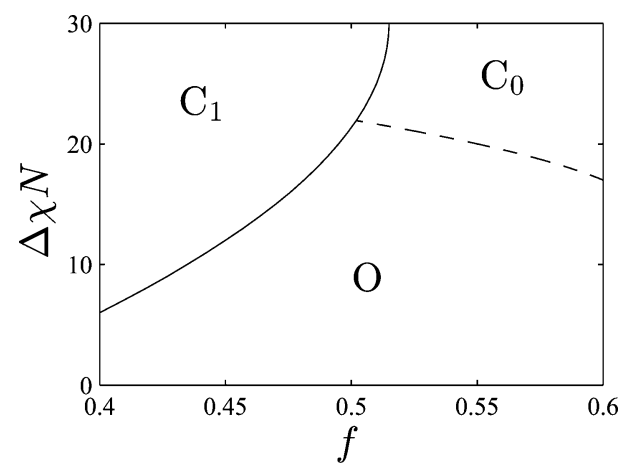

Figure 5. Phase diagram for the step edge separating the $L_{2}^{\|}$and $L_{4}^{\|}$ phases. The solid and dashed curves denote discontinuous and continuous transitions, respectively.

of the bilayer to bend more easily, which helps the A-rich surface domains join together. At large $\Delta \chi N$, the $\mathrm{C}_{0}$ morphology switches to the $C_{1}$ morphology when $f \lesssim 0.5$. This is because the bilayer prefers the minority component in its core due to the curvature of the $\mathrm{A} / \mathrm{B}$ interface along its edge and the general preference for the shorter blocks to be on the inside of any interfacial curvature. ${ }^{25,36}$ Because the closed $\mathrm{C}_{0}$ and $\mathrm{C}_{1}$ morphologies are topologically distinct, they are separated by a discontinuous transition, which we denote by a solid curve. Although it is a thermodynamic transition within mean-field theory producing a singularity in the free energy, thermal fluctuations will technically destroy (or smear) the transition since the step edge is a one-dimensional structure. ${ }^{35}$

Adding another monolayer to the film results in a step edge between the $\mathrm{L}_{3}^{\|}$and $\mathrm{L}_{5}^{\|}$phases. At large $f$, where the core of the bilayer prefers the B (blue) component, there are now two competing closed morphologies, $\mathrm{C}_{0}$ and $\mathrm{C}_{2}$, depicted in Figure 6. Notice that the domains of the $\mathrm{C}_{2}$ morphology are much more uniform in thickness. It is well understood that variations in domain thickness are indicative of packing frustration, ${ }^{19,36}$ and indeed $\mathrm{C}_{2}$ has a lower free energy than $\mathrm{C}_{0}$.

Figure 7 shows the phase diagram for the different step edges between the $L_{3}^{\|}$and $L_{5}^{\|}$phases. In this case, the continuous transition from $\mathrm{O}$ to $\mathrm{C}_{0}$ is preempted by the more stable $\mathrm{C}_{2}$ morphology, which makes the $\mathrm{O}$ region even smaller than before. As a result, all the transitions in the phase diagram are
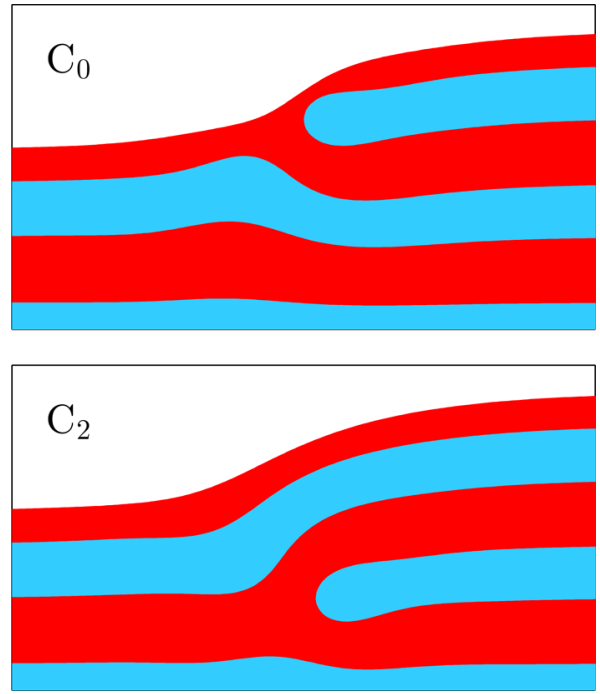

Figure 6. Closed $\mathrm{C}_{0}$ and $\mathrm{C}_{2}$ morphologies of the step edge separating the $\mathrm{L}_{3}^{\|}$and $\mathrm{L}_{5}^{\|}$phases, calculated for $\Delta \chi N=30$ and $f=0.55$. Each image is $8 \times 4.5$ in units of $a N^{1 / 2}$.

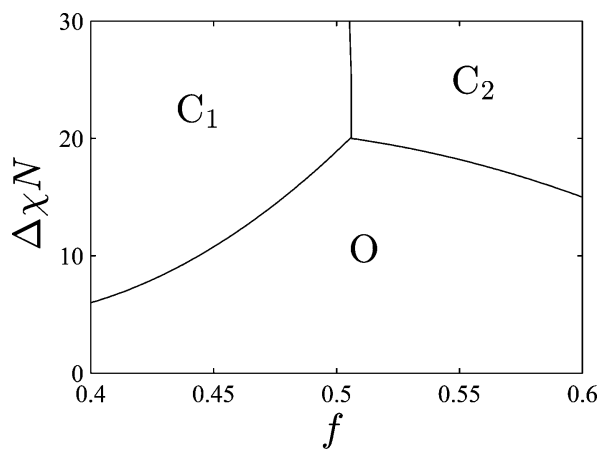

Figure 7. Phase diagram for the step edge separating the $L_{3}^{\|}$and $L_{5}^{\|}$ phases. The solid curves denote that the transitions are discontinuous.

now discontinuous. On the basis of our simple argument regarding interfacial curvature, the boundary between $C_{1}$ and $\mathrm{C}_{2}$ should occur along the symmetry line $f=0.5$. The fact it occurs at slightly larger $f$ indicates that $\mathrm{C}_{1}$ has less packing frustration than $\mathrm{C}_{2}$.

For our final example, we add three more monolayers to the film, which produces a step edge separating the $\mathrm{L}_{6}^{\|}$and $\mathrm{L}_{8}^{\|}$ phases. Figure 8 shows the potential locations of the semiinfinite bilayer among the closed $\left(\mathrm{C}_{\mu}\right)$ morphologies. Given that the minority component must occupy the bilayer core, the three morphologies on the left $(\mu=0,2$ and 4$)$ are the most competitive for $f \gtrsim 0.5$. However, it is the $\mathrm{C}_{2}$ morphology that has the lowest free energy, because the domain structure around the edge of the semi-infinite bilayer is able to adopt a more ideal shape for minimizing packing frustration if the bilayer is near the center of the film. When $f \lesssim 0.5$, the three morphologies on the right $(\mu=1,3$, and 5) are favored. In this case, $C_{5}$ is significantly less stable than $C_{1}$ and $C_{3}$, but our numerical accuracy is not sufficient to identify which of the latter two has the lowest free energy. Although there is an advantage of placing the semi-infinite bilayer toward the center of the film, which favors $\mathrm{C}_{3}$, all the monolayers above the bilayer need to bend around its edge, which disfavors $\mathrm{C}_{3}$.

The phase diagram for our thickest film is plotted in Figure 9. As before, the increase in film thickness favors the closed $\left(\mathrm{C}_{\mu}\right)$ 

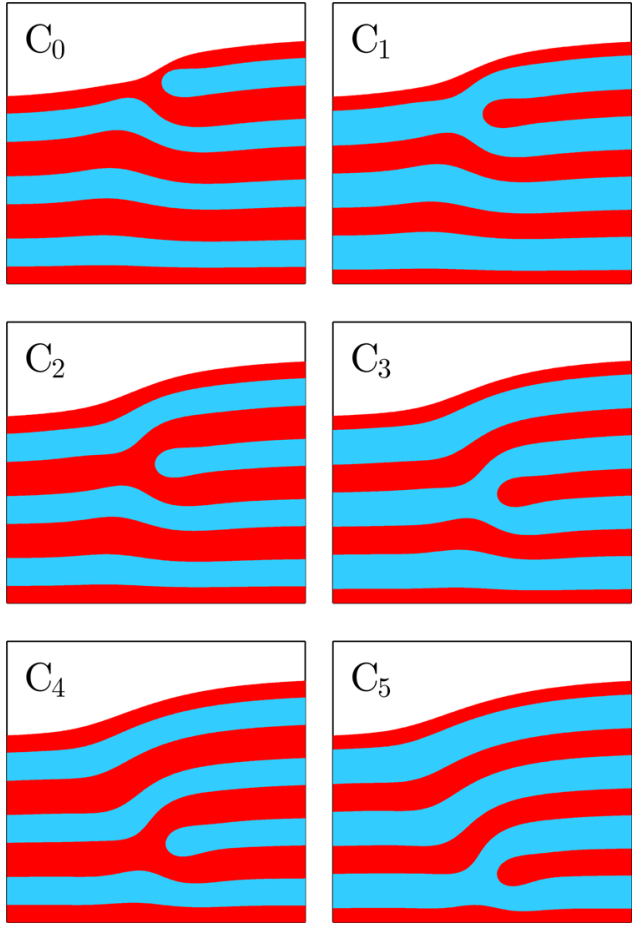

Figure 8. Closed $\mathrm{C}_{\mu}$ morphologies of the step edge separating the $\mathrm{L}_{6}^{\|}$ and $\mathrm{L}_{8}^{\|}$phases, calculated for $\Delta \chi N=30$ with $f=0.55$ and 0.45 for even and odd $\mu$, respectively. Each image is $8 \times 7.5$ in units of $a N^{1 / 2}$.

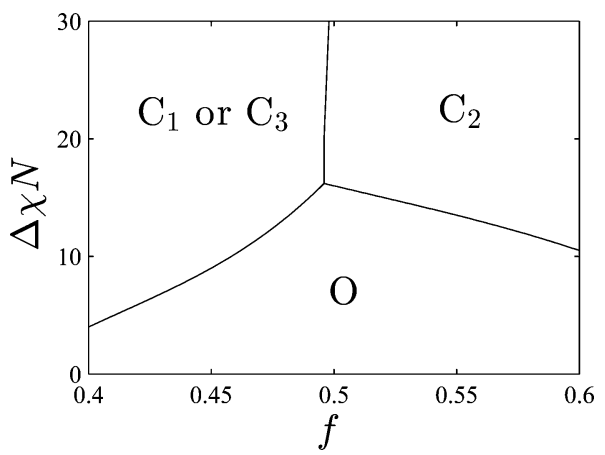

Figure 9. Phase diagram for the step edge separating the $L_{6}^{\|}$and $L_{8}^{\|}$ phases. The solid curves denote that the transitions are discontinuous.

morphologies at the expense of the open $(\mathrm{O})$ morphology. This is primarily because the bilayer in the $\mathrm{C}_{\mu}$ morphologies is further from the surfaces of the film, which helps reduce packing frustration. Indeed, the forked A-rich (red) domain of the $\mathrm{C}_{2}$ morphology in Figure 8 has a smooth symmetrical shape, whereas it is distorted in the thinner film of Figure 6 by its proximity to the substrate. Also notice that for the thicker film where the shape of the forked domain is less affected by the surfaces of the film, the transition between odd and even $\mu$ occurs closer to the symmetry line, $f=0.5$.

Line Tension. The main contribution to the free energy, $F$, of our system comes from the coexisting $\mathrm{L}_{\nu}^{\|}$and $\mathrm{L}_{\nu+2}^{\|}$phases, but there is also a small excess free energy, $\Delta F$, associated with the step edge. Naturally, $\Delta F$ is proportional to the length of the step edge, $d_{y}=V / d_{x} d_{z}$, and therefore its energetic cost is expressed as a line tension $\Gamma=\Delta F / d_{y}$. To determine the excess free energy, we perform the double-tangent construction ${ }^{20}$ illustrated in Figure 10. This is done by first solving the SCFT for the $L_{\nu}^{\|}$and $L_{\nu+2}^{\|}$phases and plotting their free energies per

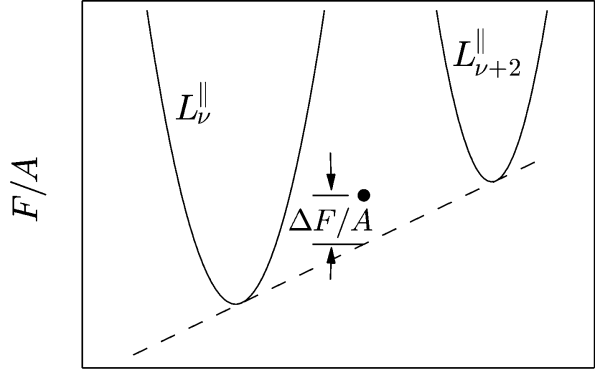

$\phi$

Figure 10. Schematic illustration of the double-tangent construction used to calculate the line tension, $\Gamma=\Delta F / d_{y}$, of a step edge. The excess free energy, $\Delta F$, is the difference between the total free energy (solid dot) and that of the coexisting $\mathrm{L}_{\nu}^{\|}$and $\mathrm{L}_{\nu+2}^{\|}$phases. The latter is given by the tangent (dashed line) to the free energies of the individual $\mathrm{L}_{\nu}^{\|}$and $\mathrm{L}_{\nu+2}^{\|}$phases (solid curves).

unit area (solid curves) as a function of the amount of diblock copolymer, $\phi$. The tangent to these curves represents the free energy of the two coexisting phases ignoring the energetic cost of the step edge, and thus $\Delta F$ is just the difference between the total free energy of the system (solid dot) and the tangent (dashed line).

Figure 11 shows the line tensions of step edges between the $\mathrm{L}_{\nu}^{\|}$and $\mathrm{L}_{\nu+2}^{\|}$phases for $\nu=1,2$ and 3, plotted as a function of

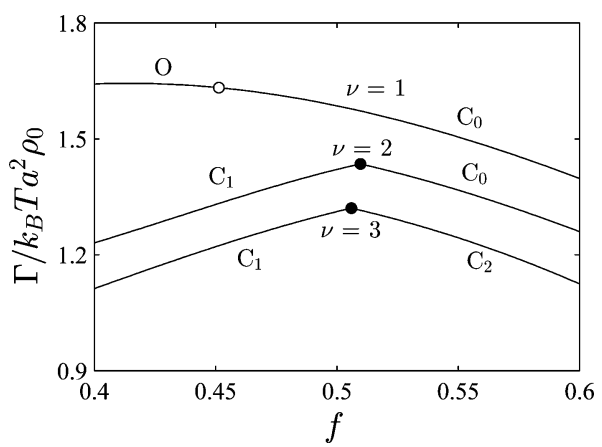

Figure 11. Line tensions, $\Gamma$, of the step edges separating the $L_{\nu}^{\|}$and $\mathrm{L}_{\nu+2}^{\|}$phases, plotted as a function of diblock composition, $f$, for a fixed surface affinity of $\Delta \chi N=25$. The open and closed dots denote continuous and discontinuous transitions, respectively.

diblock composition, $f$, at a surface affinity of $\Delta_{\chi} N=25$. The dots denote transitions between the different morphologies. For the thinnest film $(\nu=1)$, there is a continuous transition between open $(\mathrm{O})$ and closed $\left(\mathrm{C}_{0}\right)$ morphologies, but as we have mentioned previously it is not an actual thermodynamic transition. For the thicker films $(\nu=2$ and 3$)$, the transitions between closed $\left(\mathrm{C}_{\mu}\right)$ morphologies are discontinuous. Although they produce kinks in the mean-field line tension indicative of true thermodynamic transitions, these would be somewhat smoothed by thermal fluctuations as explained earlier. In any case, there is a general reduction in $\Gamma$ with increasing $\nu$, which reaffirms our assertion that the edge of the bilayer causes less packing frustration in the thicker films.

AFM Measurements. The AFM phase image in Figure 12a shows a series of $\mathrm{L}_{\nu}^{\|}$phases (i.e., terraces) produced by a gradual increase in film thickness from left to right. The system selects odd values of $\nu$ due to the asymmetric wetting conditions, resulting in the sequence $\mathrm{L}_{1}^{\|}, \mathrm{L}_{3}^{\|}, \mathrm{L}_{5}^{\|}, \mathrm{L}_{7}^{\|}$and $\mathrm{L}_{9}^{\|}$. 


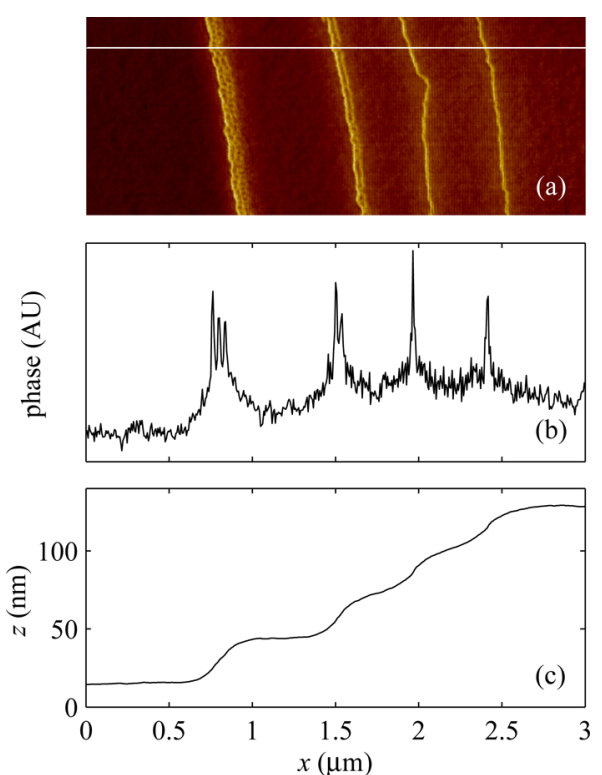

Figure 12. (a) AFM phase image of a thin diblock copolymer film. Below is the (b) phase and (c) height plotted from a single scan line across the film (the white line in the 2-d image).

As illustrated in Figure 12b, the AFM phase signal provides excellent contrast for the step edges that separate terraces, because of its sensitivity to sudden height variations and differences in the material properties of the two blocks. ${ }^{12}$ The height profile shown in Figure 12c confirms that each of the four bright lines corresponds to a single step of one bilayer $\left(L_{0}\right.$ $\approx 30 \mathrm{~nm}$ ). Interestingly, there are distinct differences among the four step edges. The first step between $L_{1}^{\|}$and $L_{3}^{\|}$is particularly broad and shows a complicated structure that varies in the lateral direction. This structure is beyond the scope of our current SCFT calculation, which assumes the morphology is uniform in the $y$ direction. Similar complex edge morphologies in the lower terraces of diblock droplets were observed previously by Carvalho and Thomas ${ }^{37}$ and by Liu et al. ${ }^{18}$ The second step from $L_{3}^{\|}$to $L_{5}^{\|}$shows two reasonably parallel lines of high intensity, with little structure in the lateral direction. By comparing the phase images obtained as the AFM rasters the image in the forward (up the film gradient) and backward (down the gradient) directions, we conclude that the dark line separating the two brighter parallel lines is consistent with PMMA interrupting the PS surface layer, or in other words an open (O) morphology. The single lines of intensity along the third step (between $\mathrm{L}_{5}^{\|}$and $\mathrm{L}_{7}^{\|}$) and fourth step (between $\mathrm{L}_{7}$ and $\mathrm{L}_{9}^{\|}$) do not indicate the presence of PMMA along the terrace boundary and can be interpreted as closed morphologies. This switch from open to closed morphologies is consistent with our phase diagrams, where the $\mathrm{O}$ region shrinks with increasing film thickness.

It is interesting to compare our experimental and theoretical surface profiles, as done in Figure 13 for a step edge between the $L_{3}^{\|}$and $L_{5}^{\|}$phases. The experimental profile is of an isolated step produced by island/hole formation, and the theoretical profile is obtained by scaling the dimensions of the SCFT calculation to match the experimental step height, $L_{0}=30 \mathrm{~nm}$. Clearly, the actual profile is far more gradual than the one predicted by SCFT, which simply implies that the actual polymer/air surface tension is much greater than that of our calculation. In other words, we would need a much larger $\chi_{\mathrm{A} h}$ in

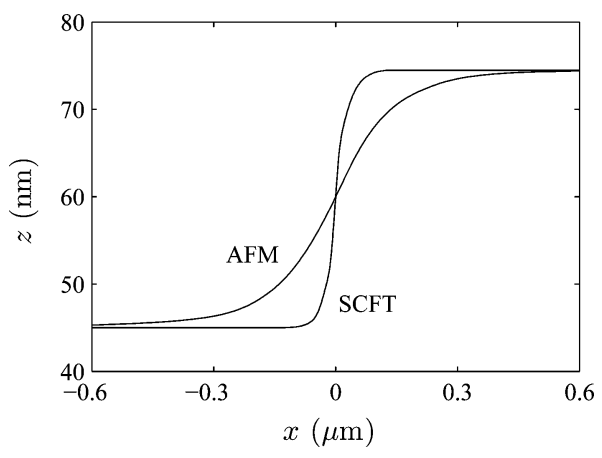

Figure 13. Comparison of the film height measured by AFM to that calculated by SCFT for an isolated step going from $L_{3}^{\|}$to $L_{5}^{\|}$.

order to quantitatively model the experimental system, which is beyond the capability of the current SCFT algorithms.

\section{DISCUSSION}

Our phase diagrams for the step edges reveal a number of general qualitative trends. When the affinity (i.e., $\Delta \chi N$ ) for A segments at the air surface is small, the step adopts an open (O) morphology where the semi-infinite bilayer resides on top of the film with the edge of its B-rich core exposed to the air. As the surface affinity increases, the top A-rich domains join together covering up the B-rich core to produce a closed $\left(\mathrm{C}_{\mu}\right)$ morphology. For films of just a few layers where the semiinfinite bilayer can only exist at the top of the film $(\mu=0)$, the transition is continuous. Otherwise, the bilayer jumps toward the center of the film $(\mu>0)$, resulting in a discontinuous transition. For asymmetric diblock copolymers, the minority component is preferred in the core of the semi-infinite bilayer due to the interfacial curvature at its edge. In our case, where the air prefers the A segments, the $\mathrm{C}_{\mu}$ morphology selects an even value of $\mu$ for $f \lesssim 0.5$ and an odd value for $f \gtrsim 0.5$.

When the film is sufficiently thick, the closed bilayer moves into the film so as to reduce the packing frustration around its edge. Although the frustration is minimized when the bilayer is at the center of the film, it is better for the bilayer to reside somewhat above center so that it has fewer monolayers on top than beneath. This is because those on top of the bilayer have to follow the curved topography of the air surface, whereas those beneath remain relatively flat. Nevertheless, in real films, it may take an extraordinary amount of time for the bilayer (or rather the dislocation caused by its edge) to migrate to its preferred location. ${ }^{17}$ Despite the fact it is a relatively simple transformation of the morphology to switch between $\mu$ and $\mu \pm$ 2 , the energetic advantage and hence driving force can be exceptionally small. Even with our relatively precise numerics, we were unable to resolve the tiny free energy difference between $\mathrm{C}_{1}$ and $\mathrm{C}_{3}$ in Figure 9 .

Of particular interest is the fact that the stability region of the open (O) morphology in the phase diagram shrinks with increasing film thickness. This happens partly because the exposed core of the semi-infinite bilayer is able to bend away from the air surface more easily in thicker films, which helps connect the top domains. The other contributing factor is the reduction of packing frustration in the closed $\left(\mathrm{C}_{\mu}\right)$ morphologies as described above, which increases their relative stability. It is the resulting shift in the $\mathrm{O}-\mathrm{C}_{\mu}$ transition that apparently triggers the switch between open and closed morphologies in Figure 12 as the film thickness increases. However, for this to be a viable explanation, the surface affinity 
in the experiment should roughly agree with our predicted location of the transition. To convert between the FloryHuggins parameters of the theory and actual surface tensions, we use the approximation $\gamma \approx k_{B} T a \rho_{0}(\chi / 6)^{1 / 2} \cdot{ }^{25}$ Taking values typical of the experiment, $T=400 \mathrm{~K}, N=500, a=6.5 \AA$, and $\rho_{0}$ $=0.006 \AA^{-3}$, the theoretical position of the transition, $\Delta \chi N \equiv$ $\chi_{\mathrm{Bh}} N-\chi_{\mathrm{Ah}} N \approx 20$, approximately equates to $\Delta \gamma \equiv \gamma_{\mathrm{PMMA}}-\gamma_{\mathrm{PS}}$ $\approx 0.7 \mathrm{dyn} / \mathrm{cm}$, which is indeed similar to the measured surface affinity of $0.25 \mathrm{dyn} / \mathrm{cm}^{38}$ As we will discuss shortly, our SCFT calculations are not sufficiently quantitative to expect better agreement than this.

The preceding study by Turner et al. ${ }^{17}$ applied a phenomenological theory to examine closed $\left(\mathrm{C}_{\mu}\right)$ morphologies. The main advantage of their calculation was its computational simplicity, which allowed them to derive some analytical predictions for certain limits. They predicted that the dislocation would tend to reside near the air surface for small surface tensions, and that it would move toward the center of the film as the tension diverged. They also found that the width of the step edge should scale as $w_{\text {step }} \sim(\gamma H)^{1 / 2}$, assuming that the tension, $\gamma$, and film thickness, $H$, are large. Although the step edges in our experiment are not sufficiently isolated to quantitatively test this prediction, we do observe a general broadening of the steps with increasing film thickness (see Figure 12c).

In order to develop their simple theory, Turner et al. ${ }^{17}$ invoked a considerable number of approximations. First, the theory was built around a function, $u(x, z)$, representing the displacement of the internal interfaces from their equilibrium positions in the absence of the extra semi-infinite bilayer. This assumption that the displacements can be represented by a continuous function is only justifiable for very thick films. Second, the extra bilayer was assumed to be a nondeformable rectangular slab. Third, their energy expression assumes $u(x, z)$ is small, which is clearly not valid in the vicinity of the defect. Fourthly, the theory ignores the free energy of the semi-infinite bilayer, and thus the calculation is unable to account for the even verses odd preference of $\mu$. Furthermore, the energy expression involves phenomenological elastic coefficients, which makes it difficult to relate their predictions to the usual diblock copolymer parameters, $f, N$, and $\chi_{\mathrm{AB}}$.

Our microscopic theory, on the other hand, is well suited to the thin films that experiments are typically concerned with. Most importantly, it places no restrictions on the size or shape of the domains, including those of the semi-infinite bilayer. As a result, it is able to account for the transformation between open $(\mathrm{O})$ and closed $\left(\mathrm{C}_{\mu}\right)$ morphologies, and it can distinguish the preference between even and odd $\mu$. As we have demonstrated, the ability to account for subtle effects such as the bending of the bilayer core is crucial in explaining the experimental results in Figure 12, where the step edges switch from open to closed morphologies with increasing film thickness.

The main limitation of our calculation is its inability to model the large polymer/air surface tensions characteristic of experiment, as illustrated by the fact the predicted surface profile is much narrower than the experimental profile in Figure 13. Given that the width of the profile increases as $w_{\text {step }} \propto \gamma^{1 / 2}$ $\propto \chi_{\mathrm{Ah}}^{1 / 4}$, it would require a tremendous increase in the FloryHuggins parameter to quantitatively match the experimental conditions. There are several practicalities preventing us from doing this. First, the Anderson mixing becomes increasingly unstable, requiring the mixing parameter to be reduced. Second, a sharper polymer profile would need a finer mesh (i.e., smaller $\Delta x$ and $\Delta z$ ). Third, a broader step would require a substantial increase in the system size (i.e., larger $d_{x}$ ). However, a detailed investigation of the Anderson mixing scheme could very well lead to a significant improvement in convergence. Of course, the numerics could be sped up by using a parallel version of the pseudospectral algorithm, ${ }^{39,40}$ and a further increase could be achieved by solving the SCFT with freely jointed chains rather than Gaussian chains. ${ }^{41}$ The key, though, may be to treat the free surface differently, such as by generalizing the fixed mask introduced earlier ${ }^{20}$ to a flexible mask.

With an improved algorithm, there would be no need to restrict our attention to steps that are uniform in the $y$ direction. We could consider more complicated morphologies like the one between the $L_{1}^{\|}$and $L_{3}^{\|}$phases in Figure 12, or the similar ones observed by Carvalho and Thomas ${ }^{37}$ and by Liu et al. ${ }^{18}$ It might also be possible to examine the interaction between the step edges in multiterraced films. This would be the final ingredient necessary to model the interesting behavior of diblock copolymer droplets ${ }^{10,24}$ and rings. ${ }^{12}$

AFM has proven to be a useful tool for probing block copolymer films, but nevertheless it only provides direct information about the surface of the film. It would be useful to have more cross-sectional TEM images. Given the theoretical ${ }^{17}$ and experimental evidence ${ }^{17,18}$ that the dislocations have a tendency to migrate toward the center of the film, the models used to explain the behavior of diblock copolymer droplets ${ }^{10,24}$ and rings ${ }^{12}$ may need to be revised.

\section{SUMMARY}

Phase diagrams were calculated for the step edges between the parallel lamellar $\left(\mathrm{L}_{\nu}^{\|}\right.$and $\left.\mathrm{L}_{\nu+2}^{\|}\right)$phases of thin films of diblock copolymer. Our attention was limited to simple morphologies, where the film has $\nu$ continuous monolayers and one semiinfinite bilayer. When the air surface has a relatively weak affinity for one of the components, an open (O) morphology occurs where the bilayer resides on top of the film with the edge of its unfavorable core exposed to the air (see Figures 1 and 2). As the affinity increases, the top domains of the $L_{\nu}^{\|}$and $\mathrm{L}_{\nu+2}^{\|}$phases join up to cover the core, resulting in a closed $\left(\mathrm{C}_{\mu}\right)$ morphology. For very thin films, where the bilayer is confined to the top of the film $(\mu=0)$, the transition occurs continuously. However, when the film is sufficiently thick, the bilayer shifts toward the center of the film, causing a discontinuous transition to a morphology with $\mu$ monolayers above the bilayer (see Figure 8). An asymmetry in the diblock causes the step to select either odd or even $\mu$, so that the minority component resides in the core of the bilayer.

The line tension of the step edge was found to decrease with increasing film thickness. This is because the packing frustration caused by the edge of the semi-infinite bilayer is shared among a greater number of monolayers. The increased thickness also allows the edge of the bilayer core to bend into the film more easily, which helps connect the top domains of the $L_{\nu}^{\|}$and $L_{\nu+2}^{\|}$ phases. This explains our experimental AFM image in Figure 12 , where the step edge between the $L_{3}^{\|}$and $L_{5}^{\|}$phases appears to be an open morphology, while the step edges between the thicker terraces resemble closed morphologies. The step edge between the $L_{1}^{\|}$and $L_{3}^{\|}$phases involves a complicated morphology, which is beyond the scope of our present calculations.

While our SCFT calculations provided qualitative predictions that can be readily understood in terms of standard block 
copolymer principles, such as spontaneous interfacial curvature and packing frustration, they were not able to quantitatively model our experimental results. In order to retain the incompressibility assumption typical of SCFT calculations, we replaced the air region by an incompatible homopolymer. This allowed the topography of the air surface to adjust freely in response to the morphology of the block copolymer film and the surface energies, which were controlled by adjusting the $\chi_{\mathrm{Ah}}$ and $\chi_{\mathrm{Bh}}$ parameters. The shortcoming of this strategy is that the numerics cannot cope with sufficiently large values of $\chi_{\mathrm{Ah}}$ and $\chi_{\mathrm{Bh}}$ to quantitatively match experimental conditions, as demonstrated in Figure 13 by the fact that the step edges are too steep. We are currently considering alternative ways of modeling the free surface, and hopefully accurate quantitative predictions will be possible in the near future.

\section{AUTHOR INFORMATION}

\section{Corresponding Author}

*E-mail: m.w.matsen@reading.ac.uk.

Notes

The authors declare no competing financial interest.

\section{ACKNOWLEDGMENTS}

This work was supported by the EPSRC (U.K.) and NSERC (Canada).

\section{REFERENCES}

(1) Matsen, M. W. Curr. Opin. Colloid Interface Sci. 1998, 3, 40-47.

(2) Fasolka, M. J.; Mayes, A. M. Annu. Rev. Mater. Res. 2001, 31, 323-355.

(3) Hamley, I. W. Prog. Polym. Sci. 2009, 34, 1161-1210.

(4) Coulon, G.; Ausserré, D.; Russell, T. P. J. Phys. (Paris) 1990, 51, 777-786.

(5) Ausserré, D.; Chatenay, D.; Coulon, G.; Collin, B. J. Phys. (Paris) 1990, 51, 2571-2580.

(6) Anastasiadis, S. H.; Russell, T. P.; Satija, S. K.; Majkrzak, C. F. Phys. Rev. Lett. 1989, 62, 1852-1855.

(7) Coulon, G.; Russell, T. P.; Deline, V. R.; Green, P. K. Macromolecules 1989, 22, 2581-2589.

(8) Coulon, G.; Collin, B.; Ausserré, D.; Chatenay, D.; Russell, T. P. J. Phys. (Paris) 1990, 51, 2801-2811.

(9) Ausserré, D.; Raghunathan, V. A.; Maaloum, M. J. Phys. II (Paris) 1993, 3, 1485-1496.

(10) Croll, A. B.; Massa, M. V.; Matsen, M. W.; Dalnoki-Veress, K. Phys. Rev. Lett. 2006, 97, 204502.

(11) Croll, A. B.; Dalnoki-Veress, K. Eur. Phys. J. E 2009, 29, 239244.

(12) McGraw, J. D.; Rowe, I. D. W.; Matsen, M. W.; Dalnoki-Veress,

K. Eur. Phys. J. E 2011, 34, 131.

(13) Maaloum, M.; Ausserré, D.; Chatenay, D.; Coulon, G. Phys. Rev.

Lett. 1992, 68, 1575-1578.

(14) Pershan, P. S. J. Appl. Phys. 1974, 45, 1590-1604.

(15) Semenov, A. E. Sov. Phys. JETP 1985, 85, 733-742.

(16) Lejcek, L.; Oswald, P. J. Phys. II (Paris) 1991, 1, 931-937.

(17) Turner, M. S.; Maaloum, M.; Ausserré, D.; Joanna, J.-F.; Kunz,

M. J. Phys. II (Paris) 1994, 4, 689-702.

(18) Liu, Y.; Rafailovich, M. H.; Sokolov, J.; Schwarz, S. A.; Bahal, S. Macromolecules 1996, 29, 899-906.

(19) Matsen, M. W. J. Phys.: Condens. Matter 2002, 14, R21-R47.

(20) Matsen, M. W. J. Chem. Phys. 1997, 106, 7781-7791.

(21) Man, X.; Andelman, D.; Orland, H. Phys. Rev. E 2012, 86, 010801.

(22) Morita, H.; Kawakatsu, T.; Doi, M. Macromolecules 2001, 34, $8777-8783$

(23) Lyakhoya, K. S.; Horvat, A.; Zvelindovsky, A. V.; Sevink, G. J. A. Langmuir 2006, 22, 5848-5855.
(24) Kim., J. U.; Matsen, M. W. Soft Matter 2009, 5, 2889-2895.

(25) Matsen, M. W. In Soft Matter: Polymer Melts and Mixtures; Gompper, G., Schick, M., Eds.; Wiley-VCH: Weinheim, Germany, 2006.

(26) Fredrickson, G. H. The Equilibrium Theory of Inhomogeneous Polymers; Oxford University Press: New York, 2006.

(27) de Gennes, P.-G. Scaling Concepts in Polymer Physics: Cornell University Press: Ithaca, NY, 1979.

(28) Ranjan, A.; Qin, J.; Morse, D. C. Macromolecules 2008, 41, 942954.

(29) Stasiak, P.; Matsen, M. W. Eur. Phys. J. E 2011, 34, 110.

(30) Anderson, D. G. J. Assoc. Comput. Mach. 1965, 12, 547-560.

(31) Thompson, R. B.; Rasmussen, K. Ø.; Lookman, T. J. Chem. Phys.

2004, 120, 31-34.

(32) Matsen, M. W. Eur. Phys. J. E 2009, 30, 361-369.

(33) Croll, A. B.; Matsen, M. W.; Shi, A.-C.; Dalnoki-Veress, K. Eur. Phys. J. E 2008, 27, 407-411.

(34) When the semi-infinite bilayer is placed next to the substrate, the edge of the bilayer core contacts the substrate. An explicit surface affinity needs to be applied in order to prevent the contact, which reflects the fact that such a morphology is energetically unfavorable.

(35) Cuesta, J. A.; Sánchez, A. J. Stat. Phys. 2004, 115, 849-893.

(36) Matsen, M. W.; Bates, F. S. Macromolecules 1996, 29, 76417644.

(37) Carvalho, B. L.; Thomas, E. L. Phys. Rev. Lett. 1994, 73, 33213324.

(38) Wu, S. J. Phys. Chem. 1970, 74, 632-638.

(39) Sides, S. W.; Fredrickson, G. H. Polymer 2003, 44, 5859-5866.

(40) Kriksin, Y. A.; Khalatur, P. G. Macromol. Theory Simul. 2012, 21, 382-399.

(41) Matsen, M. W. Macromolecules 2012, 45, 8502-8509. 